\title{
Modification in Surgical Plan following Intraoperative Detection of Co-existent Right Atrial Thrombus by Transesophageal Echocardiography in Chronic Constrictive Pericarditis
}

\author{
${ }^{1}$ Rajarajan Ganesan, ${ }^{2}$ Bhupesh Kumar, ${ }^{3}$ Ganesh K Munirathinam, ${ }^{4}$ Imran Bhat, ${ }^{5}$ Sachin Mahajan
}

\begin{abstract}
Introduction: Chronic constrictive pericarditis (CCP) is common in developing countries, tuberculosis being the most common cause. Dyspnea and congestive symptoms are the most common nonspecific presenting symptoms that require further evaluation for clinching the diagnosis. Coexistent right atrial (RA) thrombus and CCP are rare occurrences. Preoperative detection of RA thrombus is very important to avoid the risk of pulmonary thromboembolism during pericardiectomy or cannulation for establishing cardiopulmonary bypass (CPB). Perioperative echocardiography may play a crucial role in this setting. We report a case of CCP in which preoperative transesophageal echocardiography (TEE) detected RA thrombus that led to change in surgical plan and provided continuous monitoring during surgery.
\end{abstract}

Case report: A 14-year-old male presented with a history of abdominal distension and pedal edema for 5 months. Physical examination revealed raised jugular venous pulse with normal heart sounds and no murmurs. Transthoracic echocardiography (TTE) revealed constrictive physiology and thickened pericardium. After induction of anesthesia, TEE revealed an irregular hyperechoic mass $(50 \times 36 \times 30 \mathrm{~mm})$ in the RA free wall that was not detected on preoperative TTE and computerized tomography (CT). Thickened pericardium all around mandated limited pericardiectomy under TEE guidance to allow bicaval cannulation for establishing CPB. This was followed by surgical removal of the thrombus and remaining pericardiectomy using CPB. His postoperative period was uneventful and the patient was discharged home on postoperative day 7 .

Conclusion: The TEE may be instrumental in diagnosis of coexistent RA thrombus in the case of $\mathrm{CCP}$ resulting in major change in surgical plan and providing perioperative monitoring to avert significant morbidity and mortality.

Keywords: Constrictive pericarditis, Pericardiectomy, Right atrial thrombus, Transesophageal echocardiography.

\footnotetext{
1,3,4 Fellow, ${ }^{2}$ Additional Professor, ${ }^{5}$ Associate Professor

${ }^{1-4}$ Department of Anaesthesia and Intensive Care, Postgraduate Institute of Medical Education and Research, Chandigarh, India

${ }^{5}$ Department of Cardiothoracic and Vascular Surgery, Postgraduate Institute of Medical Education and Research, Chandigarh, India

Corresponding Author: Bhupesh Kumar, Additional Professor Department of Anaesthesia and Intensive Care, Postgraduate Institute of Medical Education and Research, Chandigarh, India Phone: +912741444, e-mail: bhupeshkr114@gmail.com
}

How to cite this article: Ganesan R, Kumar B, Munirathinam GK, Bhat I, Mahajan S. Modification in Surgical Plan following Intraoperative Detection of Co-existent Right Atrial Thrombus by Transesophageal Echocardiography in Chronic Constrictive Pericarditis. J Perioper Echocardiogr 2017;5(1):34-37.

\section{Source of support: Nil}

Conflict of interest: None

\section{INTRODUCTION}

Chronic constrictive pericarditis is relatively common in developing countries, tuberculosis being the most common cause. ${ }^{1}$ Dyspnea and congestive symptoms are common nonspecific presenting symptoms that require further evaluation for clinching the diagnosis. Conventional CT scanners are used to evaluate circumferential pericardial thickness and are considered superior to TTE for surgical planning. Pericardial thickness measured by TEE has been shown to be comparable with that measured by CT with the added advantage of evaluation of cardiac function and associated lesion. ${ }^{2}$ Coexistent RA thrombus and CCP are rare, ${ }^{3}$ the preoperative detection of which is very important to avoid the risk of pulmonary thromboembolism during pericardiectomy or cannulation for establishing CPB. We report a case of CCP in which preoperative TEE detected RA thrombus that led to change in surgical plan and provided for continuous monitoring during surgery.

\section{CASE REPORT}

A 14-year-old male presented with history of abdominal distension and pedal edema for 5 months. Clinical examination revealed raised jugular venous pulsation with normal heart sounds and no murmurs. Electrocardiogram showed sinus rhythm. Preoperative TTE revealed thickened pericardium $(6 \mathrm{~mm})$, dilated inferior vena cava (IVC; $21 \mathrm{~mm}$ ) with no respiratory variation and constrictive physiology $(30 \%$ variation in peak mitral inflow velocity and lateral wall tissue Doppler velocity of $10.8 \mathrm{~cm}^{-1}$ ). The CT showed thickened pericardium with no features of intracardiac thrombus. He was planned for pericardiectomy. 


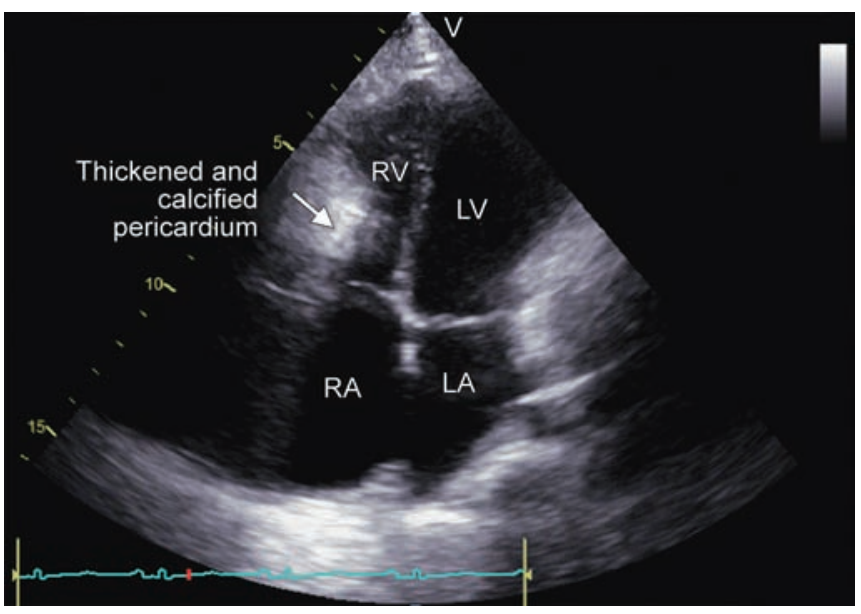

Fig. 1: Transthoracic apical four-chamber view showing the thickened pericardium with calcium plaques obscuring the full view of right atrium. LA: Left atrium; LV: Left ventricle; RA: Right atrium; $\mathrm{RV}$ : Right ventricle

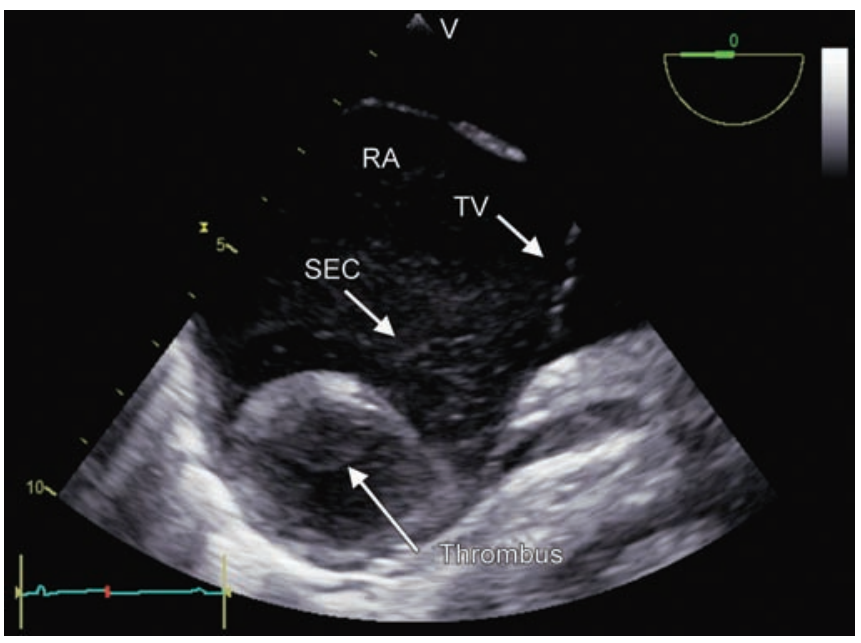

Fig. 3: Transesophageal modified four-chamber view showing a thrombus and spontaneous echo contrast in the right atrium; SEC: Spontaneous echo contrast; TV: Tricuspid valve

In the operative room, repeat TTE confirmed the findings of preoperative TTE and no additional finding was noted (Fig. 1, Video 1). The TEE done after induction of general anesthesia and institution of positive pressure ventilation confirmed thickened and calcified pericardium with a maximum thickness of $12 \mathrm{~mm}$. Lateral part of atrioventricular grove and adjacent right ventricle (RV) free wall appeared abnormally bulging in the RV cavity (Fig. 2, Video 2). The RV free wall motion was restricted, while left ventricle function appeared normal. There was mild tricuspid regurgitation. Doppler examination across mitral inflow showed 30 and $23 \%$ respiratory variations in peak $\mathrm{E}$ wave velocity and velocity time integral respectively. Spontaneous echo contrast was visualized in the right atrium and IVC was dilated $(21 \mathrm{~mm})$. Additionally, an irregular hyperechoic mass $(50 \times 36 \times 30 \mathrm{~mm})$ was visualized in the RA arising from its free wall with a broad base (Fig. 3, Video 3). The mass

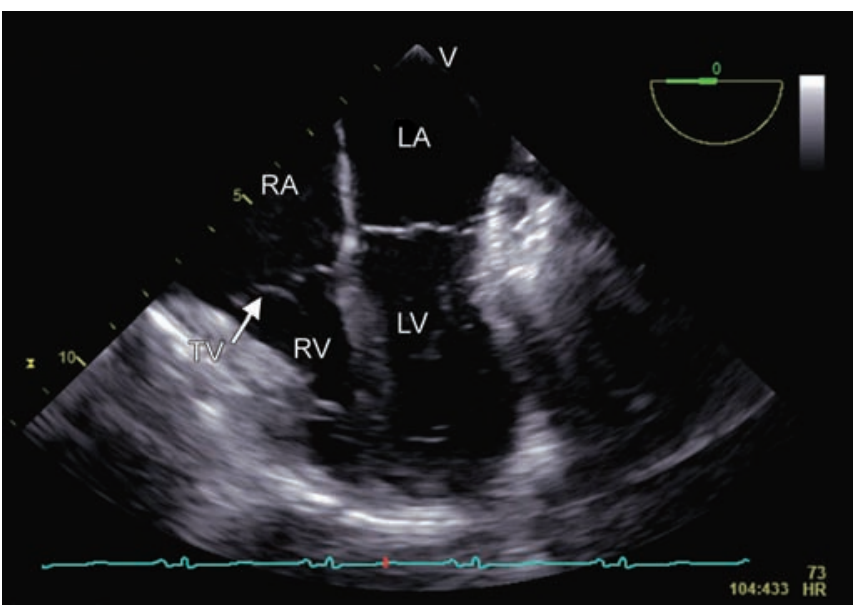

Fig. 2: Transesophageal midesophageal four-chamber view showing thickened pericardium and bulging in of RV free wall below atrioventricular groove. TV: Tricuspid valve; LA: Left atrium; LV: Left ventricle; RA: Right atrium; RV: Right Ventricle

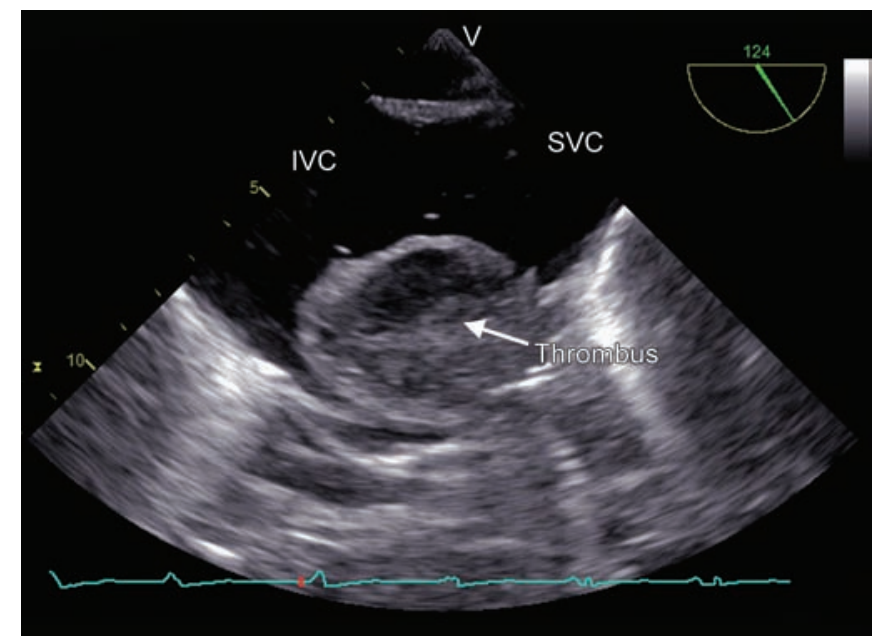

Fig. 4: Transesophageal bicaval view showing the thrombus away from the SVC and IVC

had homogeneous texture and was located away from the tricuspid valve, coronary sinus, and the superior vena cava (SVC) and IVC (Fig. 4). Continuous wave Doppler across tricuspid annulus showed minimal gradient (mean gradient $1 \mathrm{~mm} \mathrm{Hg}$ ).

Following this finding on TEE, the surgical plan was changed to pericardiectomy using $\mathrm{CPB}$ and excision of RA thrombus. Intraoperative surgical finding confirmed the presence of thickened pericardial band around right atrioventricular groove and adjacent RV free wall (Fig. 5). With TEE guidance and minimal manipulation of heart, limited pericardiectomy around SVC and IVC was performed to institute bicaval cannulation for $\mathrm{CPB}$. The right atrium was opened and the thrombus was removed. Total $\mathrm{CPB}$ duration was 20 minutes. Patient was weaned off $\mathrm{CPB}$ with moderate doses of inotropes. Remaining part of pericardiectomy was completed after termination of CPB. Patient was shifted to intensive care unit (ICU) in a 

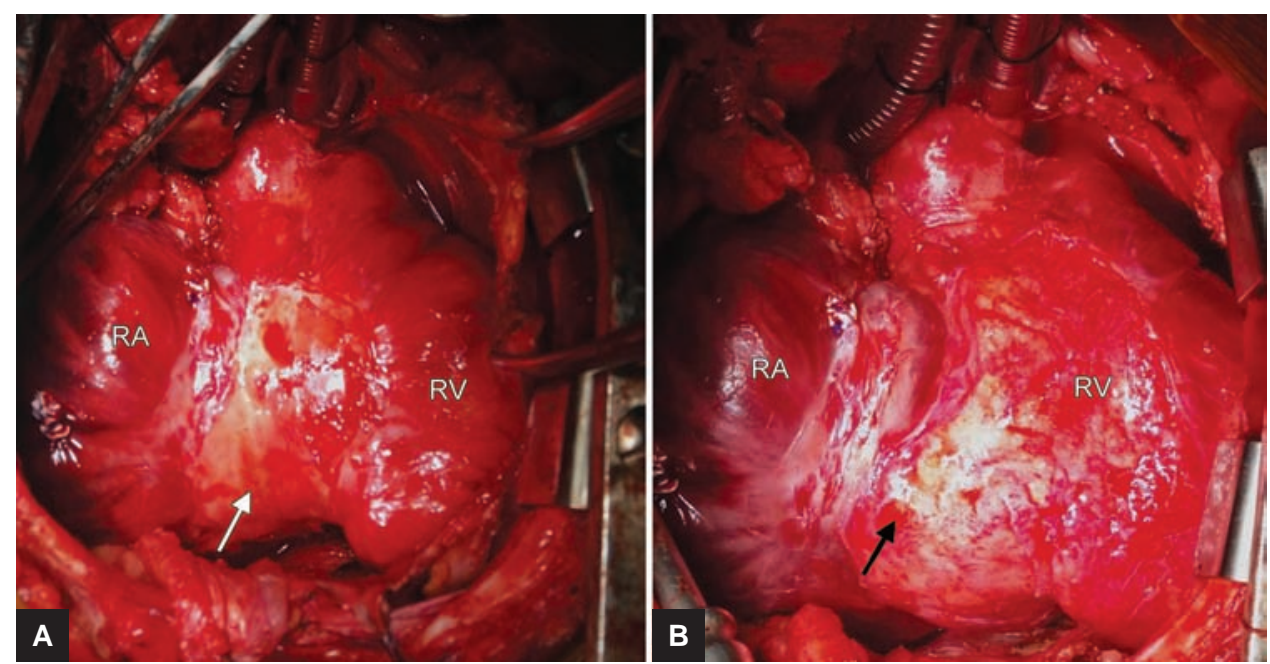

Figs 5A and B: Intraoperative surgical finding: (A) Thickened band (white arrow) of pericardium at right atrioventricular groove and adjacent RV free wall; (B) Bulging of RV after resection of thickened pericardial band (black arrow)

hemodynamically stable condition for elective mechanical ventilation. Patient's trachea was extubated next day morning. Following an uneventful 3-day course in the ICU, he was shifted to ward and finally discharged from the hospital in a stable condition.

\section{DISCUSSION}

The association of RA thrombus with CCP was first reported in 1987, following detection of a calcified thrombus on $\mathrm{CT} .{ }^{4} \mathrm{The}$ thrombus formation was postulated to be due to intracardiac blood stasis secondary to decreased diastolic compliance and atrial fibrillation. Endocardial damage accompanying the pericarditis was also cited as a reason in further reports. ${ }^{5,6}$ Later tricuspid valve obstruction by the thrombus causing hemodynamic collapse during mobilization of the heart, ${ }^{7}$ systemic venous thrombosis, ${ }^{3}$ pulmonary and systemic embolism ${ }^{8}$ in setting of CCP have also been described. In a study, spontaneous echo contrast was observed in all patients in atrial fibrillation with associated intracardiac thrombi. ${ }^{9}$ Index patient developed thrombosis in the absence of atrial fibrillation, most probably because of the venous stasis caused by the constrictive physiology and narrowing of tricuspid valve annulus and adjacent RV free wall due to constriction band formed by thickened pericardium around atrioventricular groove.

Right heart thrombi detected on echocardiography are of two types. Type I thrombus with long, thin, extremely mobile thrombi are mostly thrombus in transit associated with high incidence of deep venous thrombosis, low incidence of thrombogenic cardiac anomalies, and early mortality in $42 \%$ of patients. ${ }^{10}$ Type II thrombus is an immobile, nonspecific clot, and is associated with early mortality in $4 \%$ of patients.
The TEE has been shown to have better sensitivity of detecting RA thrombi than TTE, especially when associated with pacemaker wires. ${ }^{11}$ It helps in the decision made to treat thrombi by determining the size of thrombus. Real-time three-dimensional TEE adds further information to the evaluation of thrombus by determining homogeneity, vascularity, calcification, necrosis and differentiates a thrombus from a tumor mass. ${ }^{12}$ The pattern of respiratory variation in mitral valve and pulmonary vein flow velocities may reverse with positive pressure ventilation in $\mathrm{CCP}^{13}$ The TEE, thus, helps in structural as well as hemodynamic assessment in CCP. In our case, preoperative TTE and CT imaging could not detect RA thrombus that was subsequently visualized on TEE. This was possibly because of the long time gap of about a month between CT study and planned surgery. Thickened calcified pericardium distorting the right atrioventricular groove might have obscured the visualization of RA thrombus when TTE was done from the apical window on the day of surgery.

Treatment of an RA thrombus depends on the etiology and morphology of thrombus. Thrombolysis is an option in cases of type I thrombi. ${ }^{14}$ Giant atrial thrombi, however, are not amenable to thrombolysis and attempt to thrombolysis may result in fatality. ${ }^{15}$ Hence, the decision for thrombolysis, anticoagulation, or surgical removal of thrombus should be based on an individual basis depending on the presentation and morphology of the thrombus and clinical condition of the patient. ${ }^{16}$ In our case, the best recourse was to surgically remove the thrombus at the time of pericardiectomy, which was accomplished on $\mathrm{CPB}$ after bicaval cannulation, as there was a high chance of dislodgment of the thrombus during RA cannulation. The TEE was instrumental in our case in the diagnosis of the thrombus and providing continuous monitoring during pericardiectomy for prevention of embolization. 


\section{CONCLUSION}

The TEE may be instrumental in diagnosis of coexistent RA thrombus in case of CCP resulting in major change in surgical plan and providing perioperative monitoring to avert significant morbidity and mortality.

\section{REFERENCES}

1. Khandaker M, Espinosa R, Nishimura R, Sinak L, Hayes S, Melduni R, Oh JK. Pericardial disease: diagnosis and management. Mayo Clin Proc 2010 Jun;85(6):572-593.

2. Ling LH, Oh JK, Tei C, Click RL, Breen JF, Seward JB, Tajik AJ. Pericardial thickness measured with transesophageal echocardiography: feasibility and potential clinical usefulness. J Am Coll Cardiol 1997 May;29(6):1317-1323.

3. Ozhan H, Okçun b, Akdemir R. Constrictive pericarditis associated with right atrial and major venous thrombosis. Acta Cardiol 2004 Aug;59(4):435-437.

4. Nishimura T, Misawa T, Park YD, Uehara T, Hayashida K, Hayashi M. Visualization of right atrial thrombus associated with constrictive pericarditis by Indium-111 oxine platelet imaging. J Nucl Med 1987 Aug;28(8):1344-1347.

5. Katagiri M, Tanabe Y, Takahashi M, Kasuya S. Right atrial thrombosis: association with constrictive pericarditis. Ann Thorac Surg 1990 Jan;49(1):145-146.

6. Priestley KA, Wallwork J, Schofield PM. Right atrial thrombus in constrictive pericarditis. Int J Cardiol 1992 Nov;37(2):256-258.

7. Noma M, Itoh $\mathrm{T}$. Constrictive pericarditis with right atrial thrombus. Circulation 1996 Oct;94(7):1786-1786.

8. Glavas Konja B, Reskovic Luksic V, Bulum J, Lovric Bencic M, Ostojic Z, Ernst A, Separovic Hanzevacki J. Constrictive pericarditis complicated with thromboembolic incidents. Cardiologia Croatica 2015 May;10(3-4):72-73.

9. de Divitiis M, Omran H, Rabahieh R, Rang B, Illien S, Schimpf R, MacCarter D, Jung W, Becher H, Lüderitz B. Right atrial appendage thrombosis in atrial fibrillation: its frequency and its clinical predictors. Am J Cardiol 1999 Nov; 84(9):1023-1028.

10. The European working group on Echocardiography, Kronik G. The European Cooperative Study on the clinical significance of right heart thrombi. Eur Heart J 1989 Dec;10(12):1046-1059.

11. Schwartzbard AZ, Tunick PA, Rosenzweig BP, Kronzon I. The role of transesophageal echocardiography in the diagnosis and treatment of right atrial thrombi. J Am Soc Echocardiogr 1999 Jan;12(1):64-69.

12. Zaragosa-Macias E, Chen MA, Gill EA. Real time threedimensional echocardiography evaluation of intracardiac masses. Echocardiography 2012 Feb;29(2):207-219.

13. Abdalla IA, Murray RD, Awad HE, Stewart WJ, Thomas JD, Klein AL. Reversal of the pattern of respiratory variation of doppler inflow velocities in constrictive pericarditis during mechanical ventilation. J Am Soc Echocardiogr 2000 Sep;13(9): 827-831.

14. Dzudovic B, Obradovic S, Rusovic S, Gligic B, Rafajlovski S, Romanovic R, Ratkovic N, Dincic D. Therapeutic approach in patients with a floating thrombus in the right heart. J Emerg Med 2012 Nov;44(2):e199-e205.

15. Ruiz-Bailén M, López-Caler C, Castillo-Rivera A, RucabadoAguilar L, Ramos Cuadra JA, Lara Toral J, Lozano Cabezas C, Fernández Guerrero JC. Giant right atrial thrombi treated with thrombolysis. Can J Cardiol 2008 Apr;24(4):312-314.

16. Rose PS, Punjabi NM, Pearse DB. Treatment of right heart thromboemboli. Chest 2002 Mar;121(3):806-814. 